\title{
Observation and measurements of vector-boson scattering with the ATLAS detector
}

\author{
Ondřej Penc, on behalf of the ATLAS collaboration \\ Institute of Physics CAS, Na Slovance 1999/2, 182 21, Prague 8, CZ \\ E-mail: ondrej.penc@cern.ch \\ February 2020 \\ Copyright 2020 CERN for the benefit of the ATLAS Collaboration. CC-BY-4.0 license.
}

\begin{abstract}
The scattering of electroweak bosons tests the gauge structure of the Standard Model and is sensitive to anomalous quartic gauge couplings. In this document, we present recent results on vector-boson scattering from the ATLAS experiment using proton-proton collisions with a center-of-mass energy of $13 \mathrm{TeV}$ at the LHC. This includes the observation of $Z Z, W Z$, and same-sign- $W W$ production via vector-boson scattering along with a measurement of $V V$ production ( $V$ denotes $W$ or $Z$ boson) in semi-leptonic final states. The results can be used to constrain new physics that manifests as anomalous electroweak-boson self-interactions. Finally, predicted cross-sections for the electroweak scattering of two same-sign $W$ bosons in association with two jets are compared for a number of generators.
\end{abstract}

\section{Introduction}

ATLAS [1], a state-of-the-art high-energy physics detectorf installed on the LHC ring at CERN, has been running for two data-taking periods and has collected enough data to probe very unique processes of the Standard Model (SM), such as vector-boson scattering (VBS). This covers interactions of massive vector bosons in all possible Mandelstam channels including a Higgs boson exchange as depicted in Figure 1. The scattered bosons carry longitudinal polarization, which makes the process sensitive to high-energy asymptotic behavior. Imperfect cancellation of the divergent scattering realizations would violate tree unitarity as a necessary condition for perturbative renormalizability. Since the electroweak (EW) bosons arise in the SM by virtue of the embedded $S U(2)$ gauge symmetry group the VBS probes the non-abelian gauge structure of the SM

$\ddagger$ ATLAS uses a right-handed coordinate system with its origin at the nominal interaction point (IP) in the center of the detector and the $z$-axis along the beam direction. The $x$-axis points from the IP to the center of the LHC ring, and the $y$-axis points upward. Cylindrical coordinates $(r, \phi)$ are used in the transverse $(x, y)$ plane, $\phi$ being the azimuthal angle around the beam direction. The pseudorapidity is defined in terms of the polar angle $\theta$ as $\eta=-\ln [\tan (\theta / 2)]$. Angular distance is measured in units of $\Delta R=\sqrt{(\Delta \eta)^{2}+(\Delta \phi)^{2}}$. 


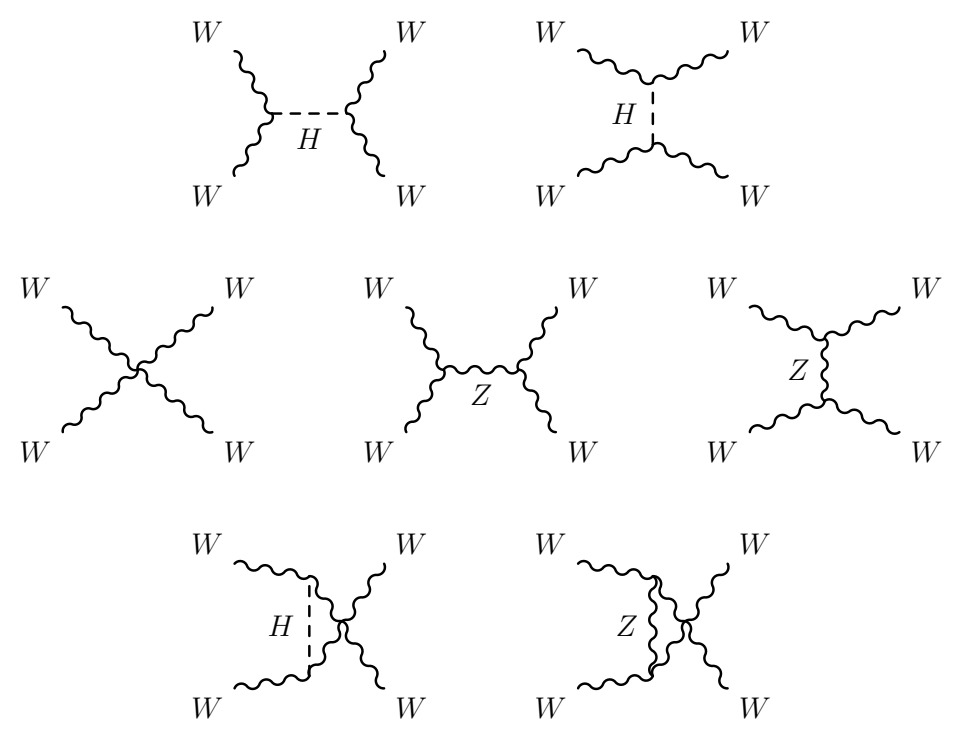

Figure 1: All tree-level Feynman diagrams realized in vector-boson scattering depicted here for the $W W \rightarrow W W$ scattering.

as well as the EW symmetry breaking. The VBS is also sensitive to physics beyond the SM which would manifest as an increase in differential cross-sections [2]. This document gives an overview of the first observations and measurements of the VBS. This can provide better understanding of the underlying physics for future theoretical interpretations and limits on possible anomalous phenomena.

The VBS signature in proton-proton collisions typically consists of at least two jets accompanying the secondary particles originating from the decay of scattered vector bosons. The di-jet system is required to be of high invariant mass and with the jets well separated in terms of rapidity, as expected for EW interacting protons. The decay mode of the bosons then classifies the studied VBS channel. The bosons can decay in several ways: hadronically to two jets, invisibly to a pair of neutrinos, visibly to two charged leptons, and semi-visibly to a charged lepton and a neutrino. All the decay combinations make a wide palette of channels to be considered in the VBS measurements. The $W W, W Z$, and $Z Z$ channels are considered with both leptonically and semi-leptonically decaying bosons. The sensitivity of the fully-hadronic channel is limited by the overwhelming background from multi-jet production and is therefore not considered.

The VBS processes form a subset in the processes with the order of EW coupling equal to six. A subset example is shown in Figure 1, while an example of non-VBS diagrams with the same order of EW coupling is depicted in Figure 2a, now including the boson decay products and accompanying quarks. The VBS processes cannot be distinguished simply from the others due to the same final state and the fact that only the total amplitude is accessible. Therefore the analyses focus on the detection and measurement of a whole class, such as the EW production of two bosons and two jets i.e. $V V j j \mathrm{EW}$ production where $V$ denotes $W^{+}, W^{-}$, and $Z$ bosons. 


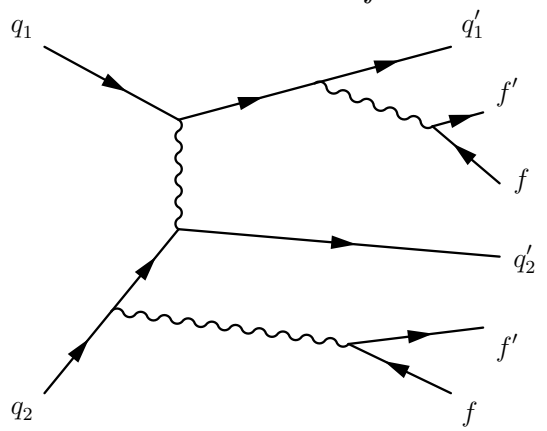

(a) $V V j j$ EW production.

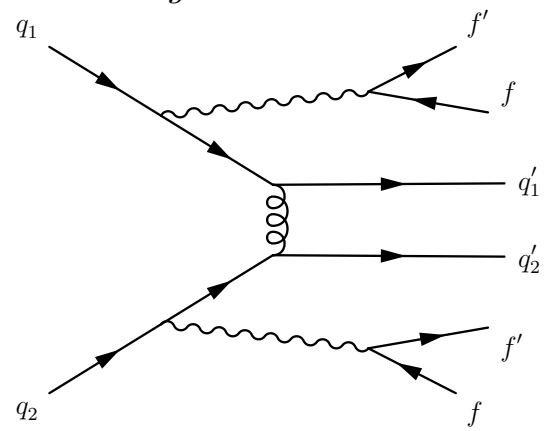

(b) $V V j j$ QCD production.

Figure 2: Typical examples of a non-VBS diagram with an order of six of EW coupling (a) and of a QCD-induced di-boson production diagram (b).

The major background for leptonic channels originates from the strong-interaction initiated processes with the same final state, typically with an order of four in EW coupling and of two in quantum-chromodynamics (QCD) coupling as shown in Figure 2b. It is common that VBS channels are categorized according to the EW-QCD production ratio. All channels have such a ratio smaller than one with one exception, which is the same-sign $W W$ channel. This extraordinary channel benefits from enhanced background suppression and was the first that brought an evidence of the VBS process already in the Run 1 of LHC [3]. The backgrounds associated to the semi-leptonic channels are dominated by $V+$ jets, while the QCD-induced background plays rather a minor role.

\section{Analysis results}

This section summarizes the VBS analyses using the ATLAS detector, with focus on fiducial phase space (PS) definitions, extracted signal strength, and measured crosssections. The main results and particular plots are emphasized at the end of each sub-section and summarized at the end of the document. For more details the reader is encouraged to refer to the original publications [4 8].

The signal and background processes are modeled using the Monte Carlo (MC) simulation. The events are passed through the GEANT 4 [9] model of the ATLAS detector [10] and then processed with the same algorithms as real data. The signal $V V j j$ samples consist of VBS processes including off-shell Higgs exchange, the other diagrams of an order of six in EW couplings, and the tri-boson production where one boson decays hadronically. The samples include the modeling of the hard scattering, parton showering, hadronization and the underlying event. The final state, in addition to the two tagging VBS jets originating from EW vertices, can consist of up to three jets stemming from initial- and final state radiation. Data-driven correction factors are applied to the physics objects to ensure that their modeling is consistent with data. 


\section{1. $W^{ \pm} W^{ \pm}$"Discovery" channel}

The first evidence of the EW production of two vector bosons and two jets was established in the $W^{ \pm} W^{ \pm} j j$ channel. The same-sign requirement suppresses processes that obey lepton number conservation and thereby significantly favors EW- against QCD-induced production.

The measurement was performed in the fiducial PS defined at the particle level in MC simulation. It is common for all of the presented analyses. The visible final state of interest consists of exactly two same-sign charged leptons originating from the boson decays and two jets as the remnants of EW interacting quarks. The charged leptons can be electrons or muons produced in the $W$ boson decay, having transverse momentum $\left(p_{\mathrm{T}}\right)$ higher than $27 \mathrm{GeV}$ and being within the ATLAS inner detector (ID) rapidity range $|\eta|<2.5$. The charged lepton is reconstructed including the fourmomenta of surrounding photons in its immediate vicinity, within a cone of radius $\Delta R=0.1$, which is called lepton dressing throughout the document. The vector sum of the transverse momenta of final state neutrinos $p_{\mathrm{T}}^{\nu \nu}$ has to be higher than $30 \mathrm{GeV}$. This

variable is later identified with $p_{\mathrm{T}}^{\text {miss }}$ in the reconstruction step.

The di-lepton system must have an invariant mass $m_{\ell \ell}$ higher than $20 \mathrm{GeV}$ and both constituents must be well separated by an angular distance $\Delta R_{\ell \ell}>0.3$ from each other.

Hadronic jets are reconstructed from all final state particles with exception of the signal leptons using the anti- $k_{T}$ algorithm [11] with radius parameter $R=0.4$. The signal jets must be in the range of $|\eta|<4.5$ with a transverse momentum $p_{\mathrm{T}}>35 \mathrm{GeV}$. Any event having a lepton overlapping with $\Delta R_{\ell j}<0.3$ in a jet is rejected.

The fiducial PS event candidate contains at least two signal jets. The leading and sub-leading jets must have $p_{\mathrm{T}}$ over 65 and $35 \mathrm{GeV}$, be separated by $\Delta y_{j j}>2$, forming a di-jet system with an invariant mass $m_{j j}$ of at least $500 \mathrm{GeV}$.

Based on statistical analysis of the reconstructed events, the background-only hypothesis is rejected with a significance of $6.5 \sigma$, corresponding to a signal strength

$$
\left.\mu_{W^{ \pm} W^{ \pm} j \text { EW }}^{\text {fid. }}=1.44_{-0.24}^{+0.26} \text { (stat. }\right)_{-0.22}^{+0.28} \text { (syst.) },
$$

which is extracted with respect to the SHERPA [12 15] fiducial cross-section prediction. The expected significance was $4.4 \sigma$. The EW production fiducial cross-section is measured using $36.1 \mathrm{fb}^{-1}$ of data to be

$$
\left.\sigma_{W^{ \pm} W^{ \pm}{ }_{j j} \text { EW }}^{\text {fid. }}=2.89_{-0.48}^{+0.51} \text { (stat. }\right)_{-0.28}^{+0.29} \text { (syst.) fb, }
$$

where the statistical uncertainty and both experimental and theoretical systematic uncertainties are included [4]. Those are considered in all analyses presented in the document.

The $W^{ \pm} W^{ \pm} j j$ channel is also of special interest in MC modeling efforts for its extraordinary EW-QCD production ratio. Moreover, the expected significance for the 


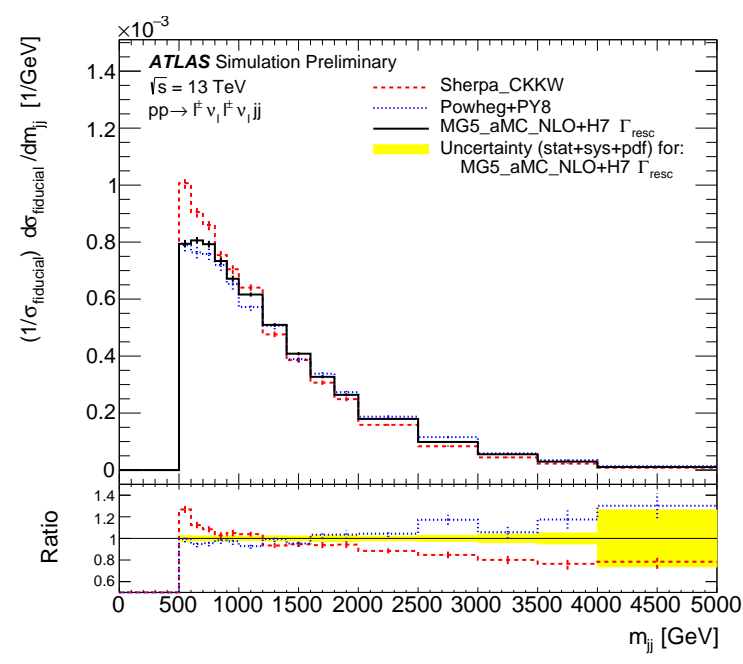

(a) Comparison of the di-jet invariant mass between three generators [5].

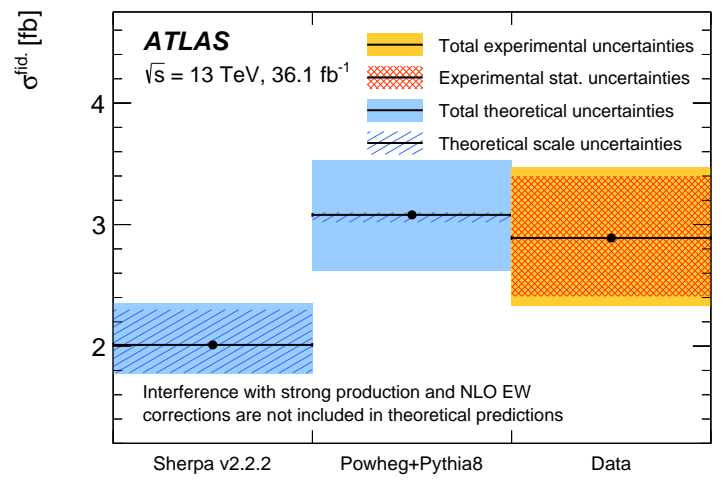

(b) Electroweak $W^{ \pm} W^{ \pm} j j$ production cross-section comparison between the data, Sherpa, and Powheg [4].

Figure 3: Illustration of the mismodeling in SHERPA, both regarding kinematic distributions (a) and the cross-section measurement (b).

signal was predicted differently as $6.5 \sigma$ by Powheg [16 19]. Such a discrepancy justifies extensive studies of the theory predictions for $W^{ \pm} W^{ \pm} j j \mathrm{EW}$ production [5]. Both cross-sections and kinematic distributions were compared, not only between the two generators but also against MADGRAPH 20]. Various setup configurations were tested including the effect of the factorization and renormalization scales. The comparison includes the parton showering tools Pythia [21], Herwig [22], and Sherpa as well as both angular ordered and dipole shower models. The study confirms a non-optimal setting of the color flow setup for parton showering in the VBS samples generated with SHERPA and explains the difference in predicted significances. In Figure 3 a comparison of all three generators reveals the discrepancy at low $m_{j j}$ in the case of SHERPA [5].

\subsection{WZ "Mix" channel}

The $W Z$ channel is studied in a final state consisting of three charged leptons and two jets. This is the only leptonic channel that mix both leptonically decaying $Z$ and $W$ bosons. Their combination brings more than one possibility to reconstruct the event out of three leptons and introduces some combinatorial background.

The fiducial PS selection requires the signal leptons to have $p_{\mathrm{T}}$ above 15 (20) $\mathrm{GeV}$ when originating from a $Z(W)$ boson decay. All signal leptons are required to be in the ID rapidity range $(|\eta|<2.5)$. The leptons are again dressed with photons within $\Delta R_{\ell \gamma}<0.1$. The invariant mass of a $Z$ boson candidate reconstructed from a same flavor opposite charge (SFOC) pair is required to be within a window of $20 \mathrm{GeV}$ centered 
around the $Z$ pole mass. The $W$ boson decay products must have a transverse mass larger than $30 \mathrm{GeV}$ defined as

$$
m_{\mathrm{T}}^{W}=\sqrt{2 p_{\mathrm{T}}^{\ell} p_{\mathrm{T}}^{\nu}\left[1-\cos \left(\Delta \phi_{\ell \nu}\right)\right]}
$$

where the $\Delta \phi_{\ell \nu}$ is the difference in azimuthal angle between the charged lepton and the neutrino transverse momentum. The charged leptons produced in the $Z$ boson decay are required to be separated by $\Delta R_{\ell_{Z, 1} \ell_{Z, 2}}>0.2$ and the distance of the lepton coming from the $W$ boson has to be $\Delta R_{\ell_{W} \ell_{z, n}}>0.3$ from each lepton of the pair.

The signal selection requests at least two jets with $p_{\mathrm{T}}>40 \mathrm{GeV}$ reconstructed with the anti- $k_{T}$ algorithm with $R=0.4$ clustering all final state particles except leptons originating from the decaying bosons. The angular isolation between a signal lepton and jet must be $\Delta R_{\ell j}>0.3$, otherwise the event is rejected.

A di-jet system is constructed from the leading jets on each side (front/back) of the detector satisfying $y_{j_{1}} \times y_{j_{2}}<0$ with an invariant mass $m_{j j}>500 \mathrm{GeV}$.

The $W Z j j$ QCD-induced production has the same final state as the signal and represents an irreducible background. Moreover, a cut-based analysis does not provide effective separation of the signal. Therefore, multivariate-analysis (MVA) techniques are employed for the signal-background discrimination by using the TMVA toolkit [22]. Discriminative variables are used as an input to the Boosted Decision Trees (BDTs). The input variables are categorized into three classes: vector-boson kinematics (boson decay products, i.e. $m_{\mathrm{T}}^{W Z}, \Delta \phi_{W Z}$ ), jet kinematics (di-jet system i.e. $m_{j j}, \Delta \phi_{j j}$ in Figure $4 \mathrm{a}$ ), and global observables (i.e. $E_{\mathrm{T}}^{\text {miss }}$, lepton centrality $\zeta_{\text {lep. }}$ ). The BDTs provide a continuous output score in the range -1 to +1 that helps discriminate the signal from the backgrounds, as shown in Figure 4b. BDT score values close to 1 indicate the maximal available separation of the signal.

The signal strength of the $W Z j j \mathrm{EW}$ production is extracted with respect to the SHERPA prediction as

$$
\mu_{W Z j j \mathrm{EW}}=1.77_{-0.40}^{+0.44}(\text { stat. })_{-0.21}^{+0.26} \text { (syst.). }
$$

The background-only hypothesis is excluded with a significance of $5.3 \sigma$ while the expected significance was $3.2 \sigma$. The fiducial $W Z j j$ EW production cross-section is measured [6] using $36.1 \mathrm{fb}^{-1}$ of data to be

$$
\left.\sigma_{W Z j j \mathrm{EW}}^{\text {fid. }}=0.57_{-0.13}^{+0.14} \text { (stat. }\right)_{-0.06}^{+0.07} \text { (syst.) fb. }
$$

\subsection{ZZ "Golden" channel}

The $Z Z j j$ channel has the smallest EW/QCD ratio. It is the rarest among all in particular with two $Z$ bosons decaying to charged leptons in the final state. Fortunately, the final state is fully reconstructible and provides the most complete information on the process. Therefore even a small number of selected events can provide a good 


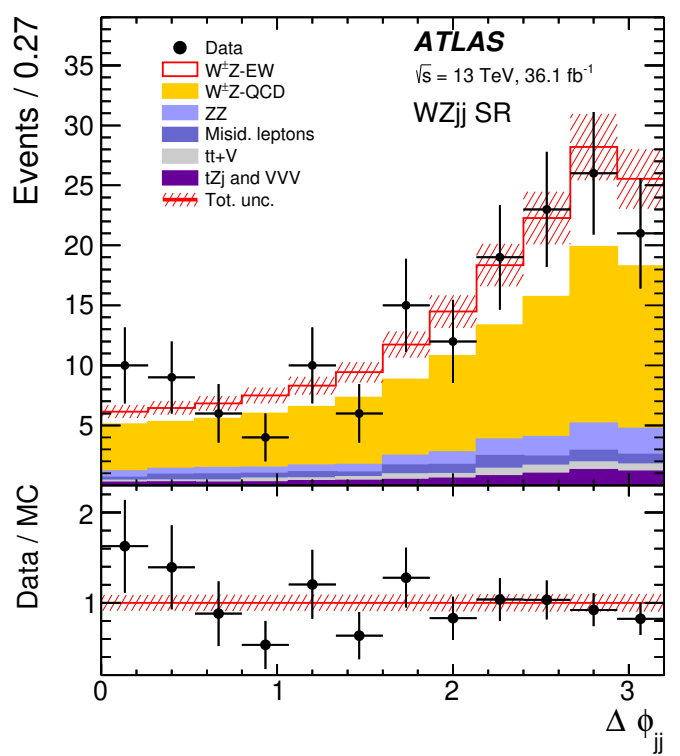

(a) Angular distance between the tagging jets in the transverse plane.

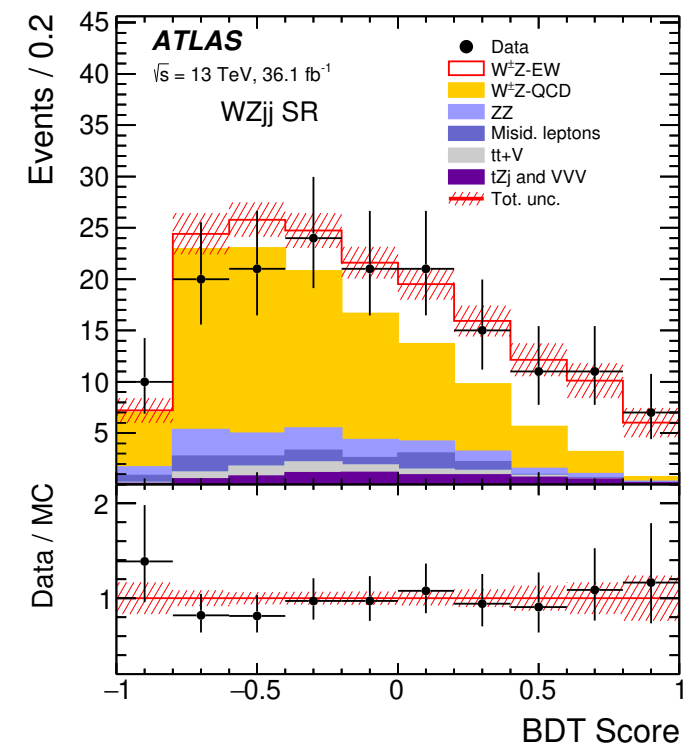

(b) Trained signal to background discriminator, BDT output.

Figure 4: BDT input observable example (a) in the signal region and BDT score (b) as output 6$]$.

understanding of the underlying physics. A final state with one $Z$ boson decaying to neutrinos is also considered. The decay channel with four neutrinos is hardly reconstructible and is not included in the analysis.

Both channels $\ell \ell \ell$ and $\nu \nu \ell \ell$ have a similar definition of the fiducial PS in which the measurement is performed, which is suitable for their subsequent combination. At the baseline object selection level, all charged leptons originating from the $Z$ boson decays must have $p_{\mathrm{T}}>7 \mathrm{GeV}$. The electrons have to be reconstructed within $|\eta|<2.47$ while muons can be up to $|\eta|<2.7(2.5)$ in the $\ell \ell \ell \ell(\nu \nu \ell \ell)$ channel. Jets are reconstructed in the same way as in the previous two analyses. The $\ell \ell \ell \ell$ channel requires jets with $|\eta|<4.5$ and $p_{\mathrm{T}}>40 \mathrm{GeV}$, however, the central jets up to $|\eta|<2.4$ are required to have $p_{\mathrm{T}}>30 \mathrm{GeV}$ only, because an advanced pileup-tagging is applied using the ID information. The $\nu \nu \ell \ell$ channel considers the full pseudorapidity range with $p_{\mathrm{T}}>60(40) \mathrm{GeV}$ for the leading (sub-leading) jet.

The event selection in the $\nu \nu \ell \ell$ channel requires a SFOC pair of leptons with $p_{\mathrm{T}}>30$ and $20 \mathrm{GeV}$ for the leading and sub-leading lepton, respectively, while vetoing a possible third one. The invariant mass of the lepton pair has to be within a window $80 \mathrm{GeV}<m_{l l}<100 \mathrm{GeV}$ and the missing transverse energy must be above $130 \mathrm{GeV}$.

After the baseline object selection, the event selection in the $\ell \ell \ell \ell$ channel considers all possible lepton quadruplets consisting of two SFOC lepton pairs with a hierarchical cut $p_{\mathrm{T}}>20,20,10 \mathrm{GeV}$ for the three leading leptons. The best quadruplet is the one minimizing the di-lepton invariant mass differences with respect to the $Z$ boson 
pole mass, defined as $\min \left(\left|m_{l^{+} l^{-}}-m_{Z}\right|+\left|m_{l^{l^{+}} l^{-}}-m_{Z}\right|\right)$. Then a requirement for the suppression of hadronic decays is imposed to all possible SFOC lepton pairs in the quadruplet by requiring $m_{l^{+} l^{-}}>10 \mathrm{GeV}$ and each lepton is required to be isolated, i.e. $\Delta R_{\ell \ell}>0.2$. Any jet in the vicinity of quadruplet leptons with $\Delta R_{\ell j}<0.2$ is removed.

An event must have at least two jets to enable reconstruction of the di-jet system out of the leading jets found on different sides of the detector with $y_{j_{1}} \times y_{j_{2}}<0$. The jets have to be separated in rapidity by $\Delta y_{j j}>2$ and the di-jet invariant mass must be $m_{j j}>300$ and $400 \mathrm{GeV}$ for the $\ell \ell \ell \ell$ and $\nu \nu \ell \ell$ channel respectively.

The MVA techniques based on a BDT classifier are again used to discriminate the signal from the backgrounds. The BDT output scores are shown in Figure 5 . The corresponding signal strength is then measured to be $1.5 \pm 0.4$ and $0.7 \pm 0.7$ for $\ell \ell \ell \ell$ and $\nu \nu \ell \ell$ channels respectively. The observed (expected) significance is $5.5(3.9) \sigma$ and $1.2(1.8) \sigma$. The combined signal strength is extracted with respect to the MADGRAPH prediction as

$$
\mu_{Z Z j j \mathrm{EW}}^{\text {fid. }}=1.35 \pm 0.34
$$

where the statistical component accounts for $88 \%$ of the total uncertainty. The combined observed (expected) significance is $5.5(4.3) \sigma$.

The combined $Z Z j j$ EW production cross-section of both channels is measured [7] using $139 \mathrm{fb}^{-1}$ of data as

$$
\sigma_{Z Z j j \mathrm{EW}}^{\mathrm{fid}}=0.82 \pm 0.21 \mathrm{fb}
$$

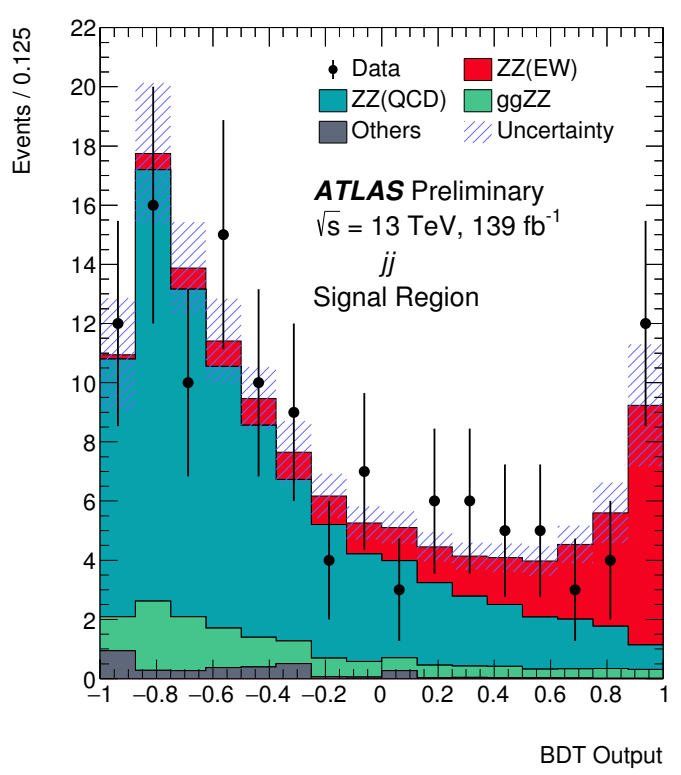

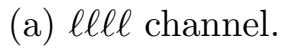

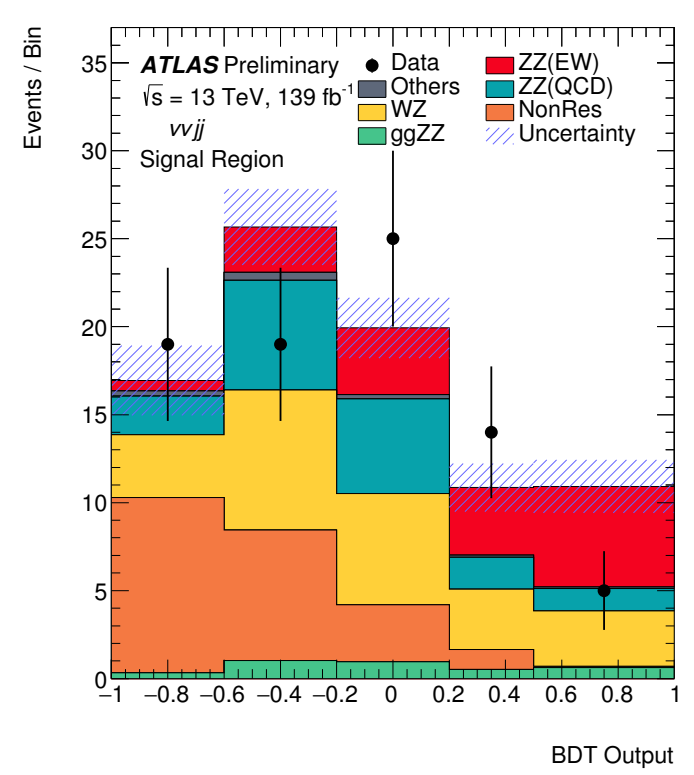

(b) $\nu \nu \ell \ell$ channel.

Figure 5: BDT score distributions in the signal region for $Z Z j j$ channels 7 . 


\subsection{Semi-leptonic channels}

The semi-leptonic VBS analysis is focused on the final states where one of the bosons decays hadronically. Together with the two VBS-tagging jets the channel considers four jets and the leptonic decay products of the other boson in the final state. From the physics point of view it consists of three channels $(W W / W Z / Z Z) j j$. From the reconstruction point of view it is more convenient to categorize the final states according to the number of leptons arising from a boson as $(Z \rightarrow \nu \nu / W \rightarrow \nu \ell / Z \rightarrow \ell \ell) V j j$ leading to zero, one, and two-lepton channels. The $n$-lepton classification remains ambiguous for the hadronically decaying boson in all channels. In addition, the zero-lepton channel includes contribution from both $W V j j$ processes. Therefore it is convenient to present the final result altogether as the $V V j j$ semi-leptonic EW production cross-section.

Multiple jets in the final state again give numerous options to reconstruct an event. Moreover, in this case there is no charge constraint and limited flavor information. Furthermore, the hadronically decaying boson can be boosted, so the two jets can merge in the detector as one large-radius jet. The baseline stage of the fiducial PS defines the objects identically for all the channels. Regular jets are reconstructed as in the previous channels using the anti- $k_{T}$ algorithm with radius parameter $R=0.4$, while the large-radius jets use $R=1.0$. The regular jets with $|\eta|<2.5$ are required to have $p_{\mathrm{T}}>20 \mathrm{GeV}$, the more forward jets up to $|\eta|<4.5$ must have $p_{\mathrm{T}}>30 \mathrm{GeV}$. The large-radius jets are considered only in the central part of the detector with $|\eta|<2.0$ and must have a high transverse momentum $p_{\mathrm{T}}>200 \mathrm{GeV}$. The leptons are required to have $|\eta|<2.5$ and $p_{\mathrm{T}}>7 \mathrm{GeV}$.

The hadronically decaying boson is reconstructed from the pool of jets either as a proper di-jet system or a large-radius jet. A function min $\left(\left|m_{j j}-m_{W}\right|,\left|m_{j j}-m_{Z}\right|\right)$ is utilized for resolved jets. For the merged case the mass of the large-radius jet $m_{J}$ is considered instead. In both cases the invariant mass of the candidate boson has to be within a mass window defined in a range $64-106 \mathrm{GeV}$. The leading jet in the resolved case must have $p_{\mathrm{T}}>40 \mathrm{GeV}$.

The leptonically decaying boson determines the category of the channel. The zerolepton channel vetoes any charged lepton and requires the neutrino pair $p_{\mathrm{T}}^{\nu \nu}>200 \mathrm{GeV}$. The one-lepton channel requires exactly one charged lepton with $p_{\mathrm{T}}^{\ell}>27 \mathrm{GeV}$ and a neutrino with $p_{\mathrm{T}}^{\nu}>80 \mathrm{GeV}$. It does not allow any $b$-jet in the jet pool to suppress the background with top quarks. The two-lepton channel demands exactly two charged leptons of the same flavor for the $Z$ reconstruction with a di-lepton invariant mass $83 \mathrm{GeV}<m_{\ell \ell}<99 \mathrm{GeV}$ and $p_{\mathrm{T}}>28(20) \mathrm{GeV}$ of the leading (sub-leading) constituent.

The VBS-tagging jets are taken from the pool of standard jets and not $b$-tagged. The di-jet system with the highest invariant mass is chosen, also demanding $m_{j j}$ to be at least $400 \mathrm{GeV}$. Both jets have to be from each side of the detector satisfying $y_{j_{1}} \times y_{j_{2}}<0$ and having $p_{\mathrm{T}}>30 \mathrm{GeV}$.

The vector boson, di-jet system, and global observables similar to the previous 
analyses are utilized as input for the training of BDTs here as well. Illustrative BDT output scores are shown in Figure 6.

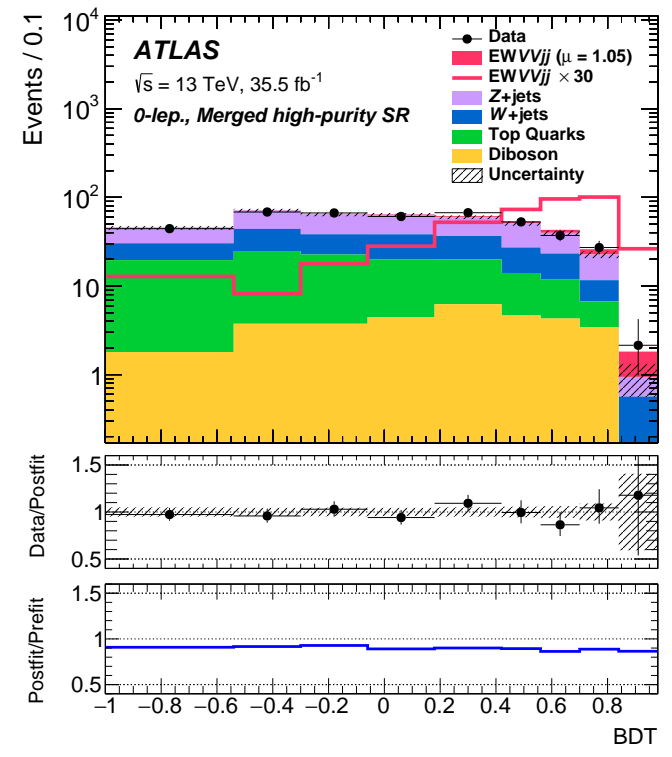

(a) Zero-lepton channel.

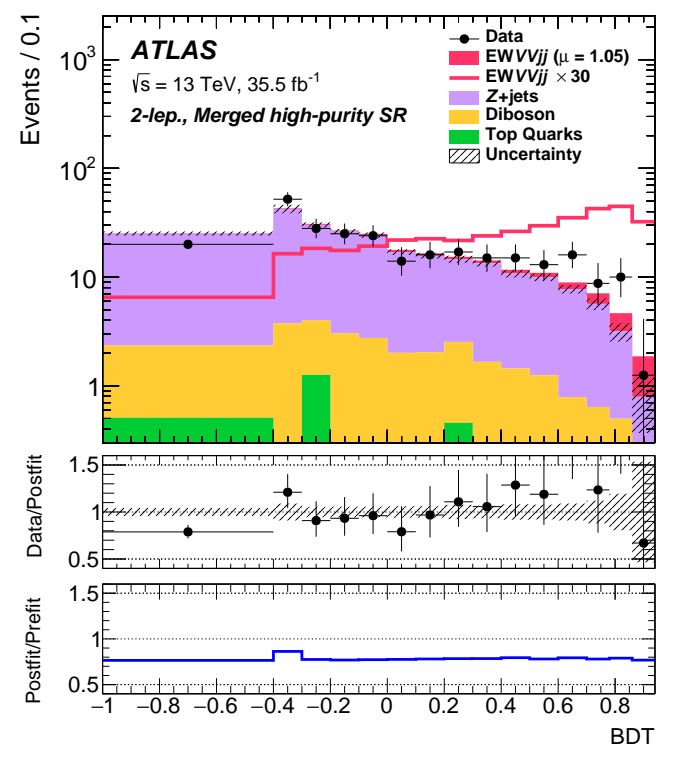

(b) Two-lepton channel.

Figure 6: Examples of BDT output scores in the signal region with a merged large-radius jet and zero (a) or two (b) leptons [8].

The signal strength is extracted with respect to the MADGRAPH prediction by the global fit based on a combination of all channels as

$$
\left.\mu_{V V j j \mathrm{EW}}^{\text {fid. }}=1.05 \pm 0.20 \text { (stat. }\right)_{-0.34}^{+0.37} \text { (syst.), }
$$

corresponding to an excess with an observed (expected) significance of $2.7(2.5) \sigma$. The fiducial $V V j j$ production cross-section is measured [8] using $35.5 \mathrm{fb}^{-1}$ of data to be

$$
\sigma_{V V j j \mathrm{EW}}^{\mathrm{fid} .}=45.1 \pm 8.6(\text { stat. })_{-14.6}^{+15.9}(\text { syst. }) \mathrm{fb} .
$$

\section{Summary}

Searches for EW di-boson production are presented in this document. All considered fully-leptonic final states exclude the background-only hypothesis with a significance of more than $5 \sigma$, opening the door for further scrutiny of the VBS phenomena. The semileptonic final states are a bit short of a $3 \sigma$ evidence, but since only $35.5 \mathrm{fb}^{-1}$ of Run 2 data were processed, it is likely that such evidence can be established when exploiting the whole dataset. A summary of signal strengths, significances, and measured crosssections in all channels is given in Table 1. The measurements are in a good agreement with the SM as the signal strengths are compatible with 1 within uncertainties. The analyses presented in this document provide very sensitive tests of the SM and also open 
Table 1: EW di-boson production summary table [4 8 ].

\begin{tabular}{l|c|c|c|c}
\hline Process & $\begin{array}{c}\text { Signal strength } \\
\sigma_{\text {data }} / \sigma_{\mathrm{MC}}\end{array}$ & $\begin{array}{c}\text { Cross-section } \\
{\left[\sigma_{E W}^{\text {fid. }}\right]=\text { fb }}\end{array}$ & $\begin{array}{c}\text { Significance } \\
\text { observed/expected }\end{array}$ & $\begin{array}{c}\text { Dataset } \\
\mathrm{fb}^{-1}\end{array}$ \\
\hline$W^{ \pm} W^{ \pm} j j\left(\nu \nu \ell^{ \pm} \ell^{ \pm}\right)$ & $1.44_{-0.24}^{+0.26}$ (stat. $)_{-0.22}^{+0.28}$ (syst.) & $2.89_{-0.48}^{+0.51}$ (stat. $)_{-0.28}^{+0.29}$ (syst.) & $6.5 / 4.4$ & 36.1 \\
$W Z j j(\nu \ell \ell \ell)$ & $1.77_{-0.40}^{+0.44}$ (stat. $)_{-0.21}^{+0.26}$ (syst.) & $0.57_{-0.13}^{+0.13}$ (stat. $)_{-0.06}^{+0.07}$ (syst.) & $5.3 / 3.2$ & 36.1 \\
$Z Z j j(\ell \ell \ell \ell \nu \nu \ell \ell)$ & $1.35_{-0.30}^{+0.30}$ (stat. $)_{-0.04}^{+0.04}$ (syst.) & $0.82_{-0.18}^{+0.18}$ (stat. $)_{-0.03}^{+0.03}$ (syst.) & $5.5 / 4.3$ & 139 \\
$V V j j$ (semi lep.) & $1.05_{-0.20}^{+0.20}$ (stat. $)_{-0.34}^{+0.37}$ (syst.) & $45.1_{-8.6}^{+8.6}$ (stat. $)_{-14.6}^{+15}$ (syst.) & $2.7 / 2.5$ & 35.5 \\
\hline
\end{tabular}

the possibility to discover new physics beyond the SM from differential cross-section distributions when an extended dataset will be used.

The fully-leptonic channels are all dominated by the statistical uncertainty. While the $W W j j$ and $W Z j j$ channels can still exploit the second half of Run 2 dataset the $Z Z j j$ needs to wait for Run 3 for more data. The semi-leptonic channel is dominated by experimental systematic uncertainties related to the multi-jet environment. The VBS physics program of Run 3 will aim at making the measurements both more precise and differential. This will enable e.g. polarization studies by using a larger dataset of proton-proton collision data.

\section{References}

[1] ATLAS Collaboration, "The ATLAS Experiment at the CERN Large Hadron Collider", JINST 3 (2008), S08003.

[2] W. Killian et al., "High-Energy Vector Boson Scattering after the Higgs Discovery", Phys. Rev. D 91 (2015), p. 096007.

[3] ATLAS Collaboration, "Evidence for Electroweak Production of $W^{ \pm} W^{ \pm} j j$ in $p p$ Collisions at $\sqrt{s}=8 \mathrm{TeV}$ with the ATLAS Detector", Phys. Rev. Lett. 113 (2014), p. 141803.

[4] ATLAS Collaboration, "Observation of electroweak production of a same-sign $W$ boson pair in association with two jets in $p p$ collisions at $\sqrt{s}=13 \mathrm{TeV}$ with the ATLAS detector", Phys. Rev. Lett. 123 (2019), p. 161801.

[5] ATLAS Collaboration, "Modelling of the vector boson scattering process $p p \rightarrow$ $W^{ \pm} W^{ \pm} j j$ in Monte Carlo generators in ATLAS", ATL-PHYS-PUB-2019-004 (2019), URL: https://cds.cern.ch/record/2655303.

[6] ATLAS Collaboration, "Observation of electroweak $W^{ \pm} Z$ boson pair production in association with two jets in $p p$ collisions at $\sqrt{s}=13 \mathrm{TeV}$ with the ATLAS detector", Phys. Lett. B 793 (2019), pp. 469-492.

[7] ATLAS Collaboration, "Observation of electroweak production of two jets in association with a $Z$-boson pair in $p p$ collisions at $\sqrt{s}=13 \mathrm{TeV}$ with the ATLAS detector", ATLAS-CONF-2019-033 (2019), URL: https://cds .cern.ch/record/ 2682845. 
[8] ATLAS Collaboration, "Search for the electroweak diboson production in association with a high-mass dijet system in semileptonic final states in $p p$ collisions at $\sqrt{s}=13 \mathrm{TeV}$ with the ATLAS detector", Phys. Rev. D 100 (2019), p. 032007.

[9] "Geant4 - a simulation toolkit", NIM A 506 (2003), pp. 250-303.

[10] ATLAS Collaboration, "The ATLAS Simulation Infrastructure", Eur. Phys. J. C 70 (2010), pp. 823-874.

[11] S. D. Ellis and D. E. Soper, "Successive combination jet algorithm for hadron collisions", Phys. Rev. D 48 (1993), pp. 3160-3166.

[12] T. Gleisberg et al., "Event generation with SHERPA 1.1", JHEP 02 (2009), p. 007.

[13] T. Gleisberg and S. Höche, "Comix, a new matrix element generator", JHEP 12 (2008), p. 039.

[14] S. Schumann and F. Krauss, "A Parton shower algorithm based on CataniSeymour dipole factorisation", JHEP 03 (2008), p. 038.

[15] S. Höche et al., "QCD matrix elements and truncated showers", JHEP 05 (2009), p. 053.

[16] B. Jäger and G. Zanderighi, "NLO corrections to electroweak and QCD production of $\mathrm{W}+\mathrm{W}+$ plus two jets in the POWHEGBOX", JHEP 11 (2011), p. 055.

[17] P. Nason, "A New method for combining NLO QCD with shower Monte Carlo algorithms", JHEP 11 (2004), p. 040.

[18] S. Frixione, P. Nason, and C. Oleari, "Matching NLO QCD computations with Parton Shower simulations: the POWHEG method", JHEP 11 (2007), p. 070.

[19] S. Alioli et al., "A general framework for implementing NLO calculations in shower Monte Carlo programs: the POWHEG BOX", JHEP 06 (2010), p. 043.

[20] J. Alwall et al., "The automated computation of tree-level and next-to-leading order differential cross sections, and their matching to parton shower simulations", JHEP 07 (2014), p. 079.

[21] T. Sjöstrand et al., "An Introduction to PYTHIA 8.2", Comput. Phys. Commun. 191 (2015), pp. 159-177.

[22] M. Bähr et al., "Herwig++ Physics and Manual", Eur. Phys. J. C 58 (2008), pp. 639-707. 